\title{
sciendo
}

\section{The Influence of Situational Variables on Ball Possession in the South African Premier Soccer League}

\author{
by \\ Alliance Kubayi ${ }^{1}$, Abel Toriola ${ }^{1}$
}

\begin{abstract}
Although the influence of ball possession in soccer has been well studied in other leagues, such information is sparse concerning the South African Premier Soccer League (PSL). The purpose of this study was to assess the impact of situational variables on ball possession in the PSL. Thirty-two matches played during the 2016-2017 PSL season were analysed using a multiple-camera match analysis system (InStat $\left.{ }^{\circledR}\right)$. Three situational variables (match outcome, match location, and quality of opposition) and team performance variables (percentage of ball possession, ball possession $<5 \mathrm{~s}$, ball possession 5-15 s, ball possession 15-45 s, and ball possession $>45 \mathrm{~s}$ ) were examined. The results showed that losing teams had the highest ball possession $(52.35 \pm 5.90 \%)$ compared to winning $(47.65 \pm 5.90 \%)$ and drawing $(50.00 \pm$ $9.98 \%)$ teams. Playing away significantly $(p<0.05)$ decreased ball possession by $5.21 \%$ compared to playing at home. Playing against weak opposition was associated with increased ball possession by $4.09 \%$. Conclusively, soccer coaches should be aware of the potential role of situational variables in determining successful team performance in a league season.
\end{abstract}

Key words: soccer, situational variables, performance indicator, ball possession.

\section{Introduction}

Over the years, performance analysis has grown drastically and has a vital role to play in the modern game of soccer (Carling et al., 2005). Preparing soccer teams to play at the professional level is a multifaceted process because it is reliant on numerous individual and team-related characteristics. For many years, the most successful soccer teams in European competitions have adopted a style of play based on ball possession (also known as an indirect playing style), allowing them to dictate the play by controlling the game (Bradley et al., 2014). This style of play is characterised by short passes and movements, playing the ball in different directions while attempting to maintain possession for longer periods of time. Teams maintain a high percentage of ball possession during a match in an attempt to enter the final third of the field to create more goalscoring chances (Bradley et al., 2014).
Research findings are inconclusive regarding the positive impact of ball possession on team success (Hughes and Franks, 2005; LagoPeñas and Dellal, 2010; Roczniok et al., 2016; Taylor et al., 2008). For example, Stanhope (2001) reported that the time spent on ball possession was not associated with team success during the 1994 FIFA World Cup. In contrast, a study conducted by Jones et al. (2004) on the 2001-2002 English Premier League season found that successful teams typically had longer ball possession than unsuccessful teams. Another study by Lago-Peñas and Dellal (2010) regarding the 2008-2009 Spanish La Liga season reported that the Barcelona Football Club had a greater percentage of ball possession $(65.29 \%)$ than teams at the bottom of the league table.

Research evidence demonstrates that variables such as a match outcome (i.e. losing, drawing or winning), match location (i.e. home or

1 - Department of Sport, Rehabilitation and Dental Sciences, Tshwane University of Technology, South Africa. 
away), and the quality of opposition (strong or weak opponents) may affect overall performance of teams (Bradley et al., 2014; Lago-Peñas and Dellal 2010; Taylor et al., 2008). With regard to the match outcome, Lago (2009) found that ball possession was significantly greater when teams were winning than losing. Concerning match location, home teams had greater possession than away teams. In fact, the authors further indicated that teams reduced their ball possession by $2.43 \%$ when playing away compared to when playing at home. Playing against strong opposition was related to a reduction in ball possession (LagoPeñas and Dellal, 2010).

To date, the effect of ball possession on performance in the South African Premier Soccer League (PSL) remains unknown. Thus, the question that arises is to what extent does the impact of situational variables influence ball possession in relation to team success in the PSL? In order to address this gap in the literature, studies are undoubtedly needed to analyse the situational variables which discriminate between teams with low and high percentages of ball possession and to examine how contextual variables may influence team performance (Bradley et al., 2014). An in-depth understanding of the situational variables affecting ball possession might fill a gap in the literature by providing soccer coaches with useful information that could be employed to devise offensive and defensive tactics for optimising overall team performance in the PSL. Consistent with Lago and Martín's (2007) suggestions, the following hypotheses were tested in this study: (1) home teams have higher ball possession compared to away teams; (2) the stronger the opponent, the greater their ball possession; and (3) teams have higher ball possession when they are losing than when they are winning.

\section{Methods}

\section{Match sample}

Thirty-two matches played during the 2016-2017 PSL season were observed using a multiple-camera match analysis system. Data were obtained from InStat ${ }^{\circledR}$, a Russia-based private company that analyses performances of soccer teams. Permission to conduct the study was granted by InStat ${ }^{\circledR}$, and ethics approval was received from the Faculty of Science Ethics
Committee of the Tshwane University of Technology, Pretoria, South Africa.

\section{Match variables}

Three situational variables (match outcome, match location, and quality of opposition) and team performance variables (percentage of ball possession, ball possession $<5 \mathrm{~s}$, ball possession $5-15 \mathrm{~s}$, ball possession $15-45 \mathrm{~s}$, and ball possession $>45 \mathrm{~s}$ ) were considered in the present study. Team's ball possession continued until an opposing player touched the ball (a shot being saved, an intercepted pass, or by means of a tackle), the referee blew the whistle for an infringement (a foul is committed or a player is in an offside position) or the ball went out of play (Jones et al., 2004; Pollard and Reep, 1997). With regard to situational variables, the match outcome was determined according to whether the team lost, drew or won; match location was categorised as either home or away (Santos et al., 2017); and the quality of opposition was based on the end-ofseason rankings of the teams (Bradley et al., 2014).

\section{Reliability testing}

The intra-observer test was used to examine the reliability of the study. The intraobserver reliability was evaluated by the author using the method of percentage error recommended by Hughes et al. (2004). Ten games were randomly selected and observed twice over a four-week period in order to avoid adverse learning effects. Consequently, the two data sets were compared and all variables were adjudged to be within the acceptable limit of $<5 \%$ (Hughes et al., 2004).

\section{Statistical analysis}

Data were reported as means and standard deviations $( \pm)$. The linear regression model was used to assess the influence of situational variables on ball possession. Negative or positive coefficients show a greater or lesser propensity to increase/decrease ball possession. The model is as follows:

Team performance $=\beta_{1}+\beta_{2}$. Match outcome $+\beta_{3}$. Match location

$$
+\beta_{4} \text {. Quality of opposition }+\varepsilon i
$$

$\beta 1$ was the intercept; $\beta 2, \beta 3$, and $\beta 4$ were the influences of all three independent variables (match location, quality of opposition, and match outcome); and $\varepsilon i$ was the disturbance term. All 
statistical analyses were conducted using the Statistical Package for the Social Sciences (SPSS), version 24 .

\section{Results}

Figure 1 showed that losing teams had the highest ball possession $(52.35 \pm 5.90 \%)$ compared to winning $(47.65 \pm 5.90 \%)$ and drawing (50.00 \pm $9.98 \%$ ) teams. Table 1 further indicated that losing teams $(3.35 \pm 2.46)$ maintained ball possession for longer periods of time than winning $(2.80 \pm 2.55)$ and drawing $(3.17 \pm 2.40)$ teams. Home teams $(50.81 \pm 7.82 \%)$ kept the ball longer than visiting teams $(49.19 \pm 7.82 \%)$. Playing against weaker opponents was associated with longer ball possession (Figure 3).

Table 2 presents the results of the analysis of the match outcome, match location, and quality of opposition in relation to ball possession. When these three predictor variables were equal to zero, ball possession was $58.81 \%$. Playing away significantly $(p<0.05)$ decreased ball possession by $5.21 \%$ compared to playing at home. Playing against weak opposition increased ball possession by $4.09 \%$. The regression model accounted for $14 \%$ of the variance in ball possession.

Table 1

Descriptive statistics on ball possession according to situational variables

\begin{tabular}{lllll}
\hline Variable & $\mathrm{BP}<5 \mathrm{~s}$ & $\mathrm{BP} 5-15 \mathrm{~s}$ & $\mathrm{BP} 15-45 \mathrm{~s}$ & $\mathrm{BP}>45 \mathrm{~s}$ \\
\hline Match outcome & & & & \\
$\quad$ Losing & $35.60 \pm 9.03$ & $50.10 \pm 9.31$ & $33.40 \pm 6.73$ & $3.35 \pm 2.46$ \\
$\quad$ Drawing & $34.04 \pm 9.44$ & $51.13 \pm 8.79$ & $33.40 \pm 6.73$ & $3.17 \pm 2.40$ \\
$\quad$ Winning & $39.30 \pm 10.91$ & $49.90 \pm 8.10$ & $31.13 \pm 8.34$ & $2.80 \pm 2.55$ \\
Match location & & & & \\
$\quad$ Home & $35.75 \pm 9.29$ & $49.66 \pm 8.76$ & $31.78 \pm 8.40$ & $3.25 \pm 2.23$ \\
$\quad$ Away & $36.59 \pm 10.60$ & $51.19 \pm 8.57$ & $30.31 \pm 7.62$ & $2.97 \pm 2.55$ \\
Quality of opposition & & & & \\
$\quad$ Stronger teams & $36.78 \pm 11.30$ & $50.28 \pm 7.78$ & $31.47 \pm 7.30$ & $2.94 \pm 2.56$ \\
$\quad$ Weaker teams & $35.56 \pm 8.40$ & $50.56 \pm 9.52$ & $30.62 \pm 8.73$ & $3.28 \pm 2.20$ \\
\hline
\end{tabular}

Table 2

The influence of situational variables on ball possession

\begin{tabular}{lccccc}
\hline Variable & BP $(\%)$ & BP $<5 \mathrm{~s}$ & BP 5-15 s & BP 15-45 s & BP $>45 \mathrm{~s}$ \\
\hline Match outcome & $-3.53(1.50)^{*}$ & $1.93(1.95)$ & $0.49(1.74)$ & $-4.03(1.49)^{* *}$ & $-0.20(0.49)$ \\
Match location & $-5.21(2.30)^{*}$ & $3.40(2.97)$ & $3.05(2.67)$ & $-6.03(2.27)^{* *}$ & $-0.60(0.75)$ \\
Quality of opposition & $4.09(2.21)$ & $-0.43(2.85)$ & $-1.67(2.56)$ & $4.37(2.18)^{*}$ & $0.06(0.71)$ \\
Intercept & $58.81(5.24)^{* *}$ & $27.87(6.78)^{* *}$ & $47.73(6.07)$ & $41.52(5.17)^{* *}$ & $4.41(1.70)$ \\
$R^{2}$ & 0.14 & 0.03 & 0.03 & 0.17 & 0.01 \\
\hline
\end{tabular}

Standard errors are in parentheses. ${ }^{* *} p<0.01 ;{ }^{*} p<0.05 . B P=$ Ball possession. $s=$ seconds 


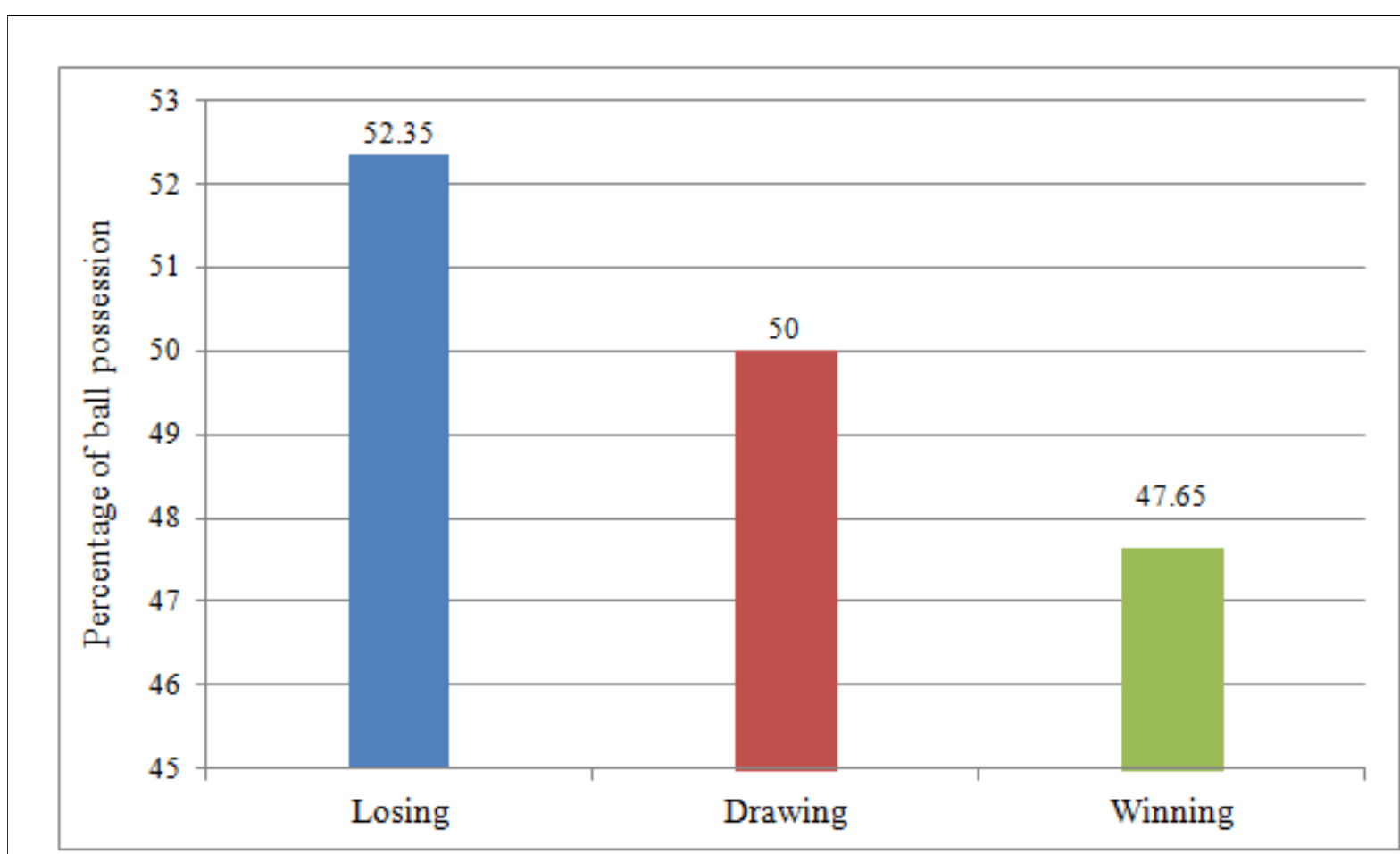

Figure 1

Ball possession according to the match outcome

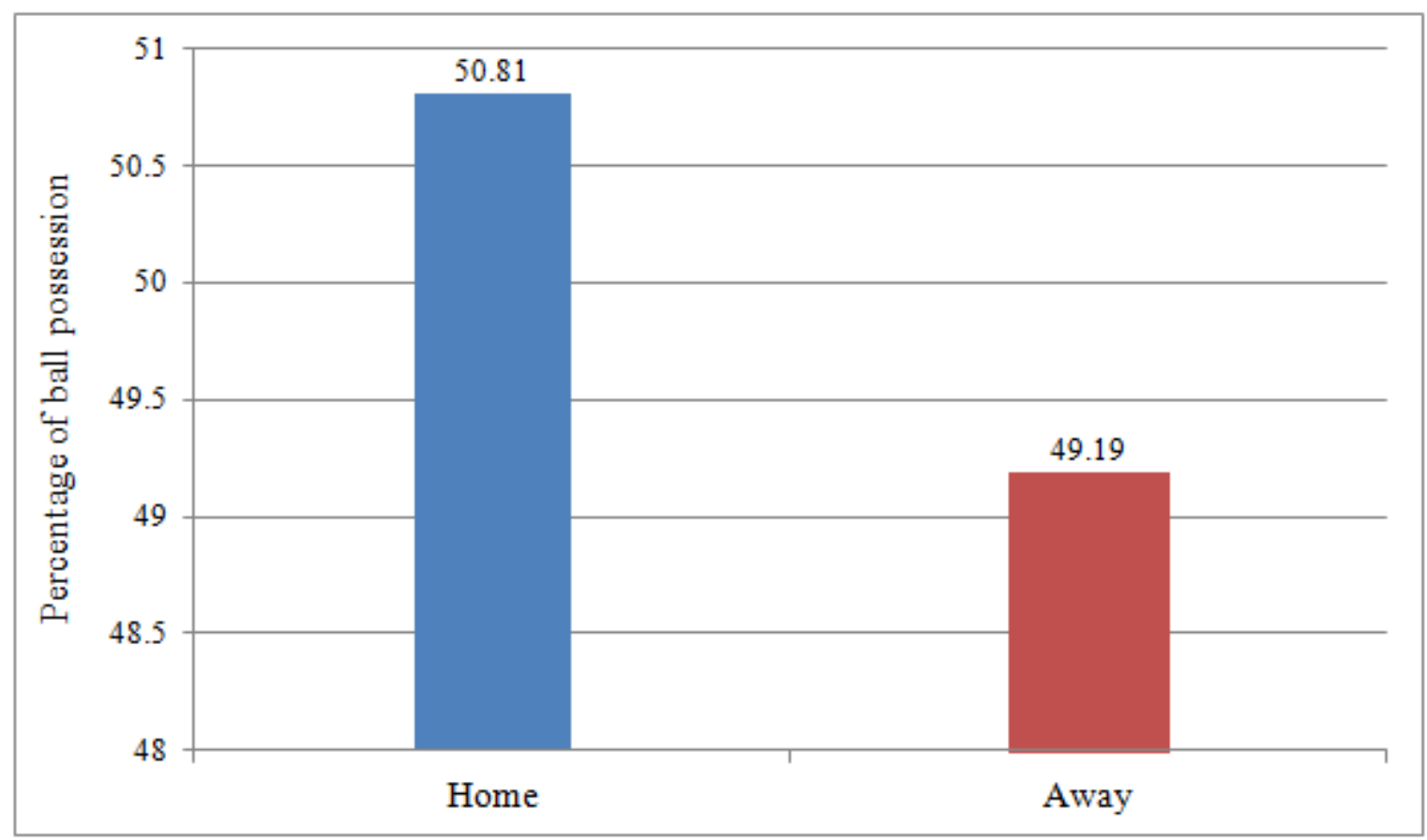

Figure 2

Ball possession based on match location 


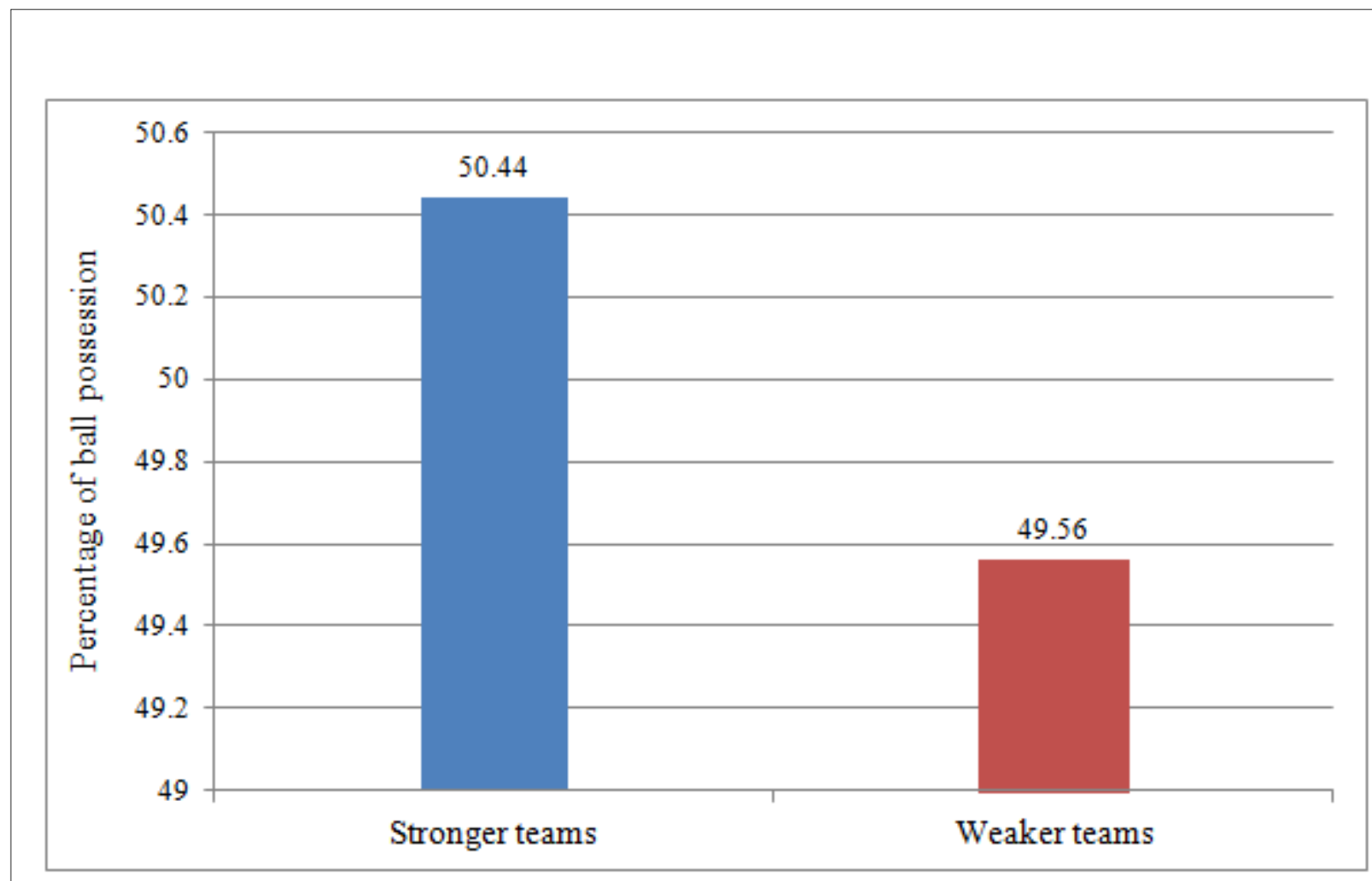

Figure 3

Ball possession according to the quality of opponents

\section{Discussion}

This study examined the effects of the match outcome, match location, and quality of opposition on ball possession among South African PSL teams. The results of the current study indicated that losing teams had a higher percentage of ball possession than winning teams. These findings are consistent with those of previous studies (e.g., Lago, 2009; Lago and Martín, 2007; Lago-Peñas and Dellal, 2010), which reported that ball possession in soccer was influenced by the scoreline. A possible explanation for this finding could be that losing teams tend to become more desperate towards the end of the match, thereby striving hard to win a game or at least settle for a draw. As a result, such teams tend to push players forward in an effort to create scoring opportunities (Reilly, 1997). This is further confirmed by Lago and Martín (2007), who reported that when a team was losing it needed to create more goal-scoring opportunities to draw or win the match, which required greater possession of the ball.

By contrast, when teams are ahead, they usually employ counter-attack tactics or a direct playing style (that is, moving the ball quickly to within scoring range, often using long passes or playing long balls downfield), thus decreasing the extent of their ball possession (Lago, 2009). Additionally, the results further showed that losing teams had longer possessions than winning teams. These findings are inconsistent with those of Jones et al. (2004), who reported that longer possession was indicative of team success. However, due to the fact that losing teams tend to have more possession of the ball in the attacking zone and less in the defensive zone (Lago, 2009), winning teams may 'park the bus' (when all players in a team play defensively, usually when a 
team intends to defend a narrow margin or at least draw the game (The Football Supernova, 2012). Consequently, this style of play could lead to players making unforced defensive errors such as kicking the ball out of play or into forward positions where they are numerically inferior, thereby decreasing time on the ball.

With regard to the quality of the opposition, stronger teams retained more possession of the ball than weaker teams. Empirical evidence has suggested that stronger opponents adopt good defensive organisational strategies, such as timely tackles and interceptions which limit opposition team's opportunities to penetrate the defence and take shots at goal (Pratas et al., 2012). Regarding match location, playing games away from home resulted in a reduction in ball possession by $5.21 \%$. This result is comparable with that of Lago-Peñas and Dellal (2010), who reported that playing away from home reduced ball possession by $2.43 \%$. This finding could be explained based on the fact that playing at home appears to give an advantage to a soccer team due to familiarity with surroundings and support from a partisan crowd. Consequently, an atmosphere created by a hostile crowd can lead to increased anxiety and bring about mistakes by visiting teams, which can affect the match outcome - in particular, by decreasing team possession (Carling et al., 2005).

In conclusion, the findings showed that when the teams played against weaker opponents, at home, and were losing, they had more ball possession. The results of this study highlight the need for soccer coaches and analysts to consider the interactive influences of the quality of opposition, match location, and match outcome when examining the technical and tactical elements of soccer performances (Taylor et al., 2008). Therefore, coaches should use such information to establish trends and objectives for teams and players in training and competition (Santos et al., 2017) in order to enhance overall performance.

\section{References}

Bradley P, Lago-Peñas C, Rey E, Sampaio J. The influence of situational variables on ball possession in the English Premier League. J Sports Sci, 2014; 32(20): 1867-1873

Carling C, Williams AM, Reilly T. Handbook of soccer match analysis: A systematic approach to improving performance. Abingdon, UK: Routledge; 2005

Hughes M, Franks I. Analysis of passing sequences, shots and goals in soccer. J Sports Sci, 2005; 23(5): 509-514

Hughes M, Cooper SM, Nevill A. Analysis of notation data: Reliability. In M Hughes, IM Franks (Eds.), Notational analysis of sport (2nd ed., pp. 189-204). London, New York, NY: Routledge; 2004

Jones PD, James N, Mellalieu D. Possession as a performance indicator in soccer. Int J Perform Anal Sport, 2004; 4: $98-102$

Lago C. The influence of match location, quality of opposition, and match outcome on possession strategies in professional association football. J Sports Sci, 2009; 27(13): 1463-1469

Lago C, Martín R. Determinants of possession of the ball in soccer. J Sports Sci, 2007; 25(9): 969-974

Lago-Peñas C, Dellal A. Ball possession strategies in elite soccer according to the evolution of the match-score: The influence of situational variables. J Hum Kinet, 2010; 25: 93-100

Pollard R, Reep C. Measuring the effectiveness of playing strategies at soccer. The Stat, 1997; 46 (4): 541-550

Pratas J, Volossovitch A, Ferreira AP. The effect of situational variables on teams' performance in offensive sequences ending in a shot on goal. A case study. The Open Sports Sci J, 2012; 5: 193-199

Reilly T. Energetics of high intensity exercise (soccer) with particular reference to fatigue. J Sports Sci, 1997; 15: 257-263

Roczniok R, Stanula A, Maszczyk A, Mostowik A, Kowalczyk M, Fidos-Czuba O, Zając A. Physiological, physical and on-ice performance criteria for selection of elite ice hockey teams. Biol Sport, 2016; 33(1): $43-48$ 
Santos P, Lago-Peñas C, García-García O. The influence of situational variables on defensive positioning in professional soccer. Int J Perform Anal Sport, 2017. In Press. doi.org/10.1080/24748668.2017.1331571.

Stanhope J. An investigation into possession with respect to time in the Soccer World Cup 1994. In MD Hughes (Ed.), Notational analysis of sport III (pp. 155-162). Cardiff: UWIC; 2001

Taylor JB, Mellalieu SD, James N, Shearer D. The influence of match location, quality of opposition and match outcome on technical performance in professional association football. J Sports Sci, 2008; 26: 885-895

The Football Supernova. The myth of 'parking the bus'. 2012. Available at http://www.thefootballsupernova.com; accessed on 14.08.2017

\section{Corresponding author:}

\section{Alliance Kubayi}

Tshwane University of Technology

Department of Sport, Rehabilitation and Dental Sciences

Republic of South Africa

Pretoria, 0001

Telephone number: +27123824272

Fax number: +27 123825801

E-mail: kubayina@tut.ac.za 